\title{
Structured Adaptive Finite-Volume Multigrid for Compressible Flows
}

\author{
H.T.M. van der Maarel ${ }^{*}$ \\ P.W. Hemker ${ }^{\dagger}$
}

\begin{abstract}
Summary
For a system of hyperbolic conservation laws, such as the Euler equations of compressible flow, in this paper we give an outline of the theory necessary to derive first and second-order accurate discretisations on a structured, adaptive finite-volume mesh. The mesh is constructed so that the equations can be defined on a rather arbitrary domain. and the usual nonlinear multigrid techniques can be used for the solution of the discrete system. During the solution process the mesh can be adapted to the solution and to the accuracy of the discrete equations. This requires a sufficiently accurate estimate of the local truncation error.

After formally introducing the geometric structure and notations, we discuss the discretisation and we study the various contributions to the local discretisation error. Emphasis is put on the discretisation involving the interfaces between the coarse and the fine parts of the grid. Our analysis leads to a small set of requirements, to be satisfied in order to attain a discretisation which is first or second-order accurate (in a sense that will be specified) with respect to the mesh size of the partitioning. Then interpolations are presented which satisfy these conditions.
\end{abstract}

\section{Introduction}

In this paper we describe the discretisation of a system of steady conservation laws in two space dimensions, using a finite volume discretisation with a structured, locally refined partitioning of the domain of definition.

We consider a system of $d$ conservation laws, defined on an open domain $\Omega \subset \mathbb{R}^{2}$, with $q: \bar{\Omega} \rightarrow \mathbb{R}^{d}, s: \Omega \rightarrow \mathbb{R}^{d}, f, g: \mathbb{R}^{d} \rightarrow \mathbb{R}^{d}$ given by

$$
\frac{\partial f(q(x, y))}{\partial x}+\frac{\partial g(q(x, y))}{\partial y}=s(x, y), \quad(x, y) \in \Omega
$$

supplemented with appropriate boundary conditions.

For the discretisation we introduce a partitioning of $\Omega$. The partitioning forms, possibly after a smooth coordinate transformation, a set of regular quadrilaterals, called

*MARIN, P.O.Box 28, 6700 AA Wageningen, The Netherlands; e-mail: maarel@marin.nl

${ }^{\dagger}$ CWI, P.O.Box 94079, 1090 GB Amsterdam, The Netherlands; e-mail: pieth@cwi.nl 
the grid. In transformed coordinates. the locally refined grid is composed of a sequence of nested grids. where each grid is a regular partitioning of a subdomain of $\Omega$. In nontransformed coordinates. the union of all quadrilaterals is an approximation of the domain of definition.

Each quadrilateral of the grid is used as a control volume on which the system of conservation laws. integrated over the control volume. is approximately satisfied. This leads to a discretisation of a weak formulation of problem (1.1).

The error in the approximation of the weak formulation consists of contributions from the various steps in the discretisation. The first step is the approximation of the domain by the partitioning. This approximation has consequences for the accuracy of the discrete equations defined for quadrilaterals along the boundary of the domain and for the "coarsegrid' approximation. The approximation of the weak formulation for each quadrilateral involves the approximation of the mean flux per unit time and 'area' across each side of each quadrilateral. The next step is the approximation of the mean flux across the sides of the quadrilaterals by the flux at the mean state at each side of a face Finally the state at the cell face is approximated from the discrete data (the available numerical solution, a cell-wise constant function). The flux may be evaluated in an upwind fashion, where each approximated flux depends in an upwind biased sense on the discrete data.

The numerical solution itself approximates the mean of the exact solution of (1.1) over each cell. Hence. with each cell is associated an approximation of the mean value on the cell of the solution of the continuous problem. This is the so-called cell-centered approach.

The decision where to refine a given composite grid (or to remove a refinement), may be based on the local discretisation error. Therefore we study the a-posteriori estimation of the local discretisation error. However. refinement should not be based solely on the local discretisation error. Apart from a sufficiently accurate discretisation scheme. an accurate solution requires that the grid provides sufficient resolution. Resolution of the grid is measured by the derivatives of the exact solution, as approximated by the numerical solution. The grid should therefore also be refined on the basis of solution gradients. For the equations associated with cells near discontinuities in the solution, an estimate of the local discretisation error is superfluous, since the grid will be refined due to the approximations of the large gradient in the solution. For the smooth part of the solution an estimate of the local discretisation error may be obtained with sufficient accuracy and can be used in a refinement strategy.

In the neighbourhood of fine-coarse grid interfaces, by nature, the discretisation scheme used is different from the one used elsewhere. Estimating the local truncation error in such a situation by means of extrapolation techniques, (as in $\tau$-extrapolation, $[3,5]$ ), requires a careful treatment of the various contributions to the local truncation error. Here we carefully consider these contributions, with emphasis on their accurate estimation.

\section{The geometric structure}

We study a system of conservation laws, defined on $\Omega$, with boundary conditions on $\partial \Omega \subset \bar{\Omega}$, where $\bar{\Omega}$ denotes the closure of $\Omega$. We assume that a rectangular domain $\tilde{\Omega} \subset \mathbb{R}^{2}$ exists and a sufficiently smooth surjective mapping $M: \overline{\widetilde{\Omega}} \rightarrow \bar{\Omega}$, which is also injective in the interior $\tilde{\Omega}$. The mapping is a transformation of the Cartesian coordinates in $\overline{\widetilde{\Omega}}$, the computational space, into Cartesian coordinates in $\bar{\Omega}$, the physical space.

As the system of conservation laws is discretised by a finite volume method, a regular 
rectangular partitioning of $\widetilde{\Omega}$ is introduced, consisting of disjoint rectangles. This defines a partitioning of $\Omega$, through the mapping $M$. A rectangle in the computational space $\widetilde{\Omega}$, as well as its image in $\Omega$. is called a cell. The partitioning is called the grid.

We consider grids on different levels of refinement. A level of refinement $l$, with $l=0,1,2, \cdots$, is a regular partitioning, denoted by $\tilde{\Omega}^{l}$, of a subdomain in $\tilde{\Omega}$, and a surjective mapping $M^{l}: \overline{\widetilde{\Omega}} \rightarrow \overline{\widehat{\Omega}}^{l}$. injective in the interior $\widetilde{\Omega}^{l}$. We use the notation $\Omega^{l}=M^{l}\left(\tilde{\Omega}^{l}\right)$ for an image under the mapping $M^{l}$. For the image $\widehat{\Omega}^{l}$ of $\tilde{\Omega}$ under $M^{l}$, and the hull $\tilde{\Omega}$ of all images of $\widetilde{\Omega}$ under the sequence of mappings $\left\{M^{l}\right\}_{l \in \mathbb{Z}}$, we use the notation

$$
\hat{\Omega}^{l}=M^{l}(\tilde{\Omega}), \text { and } \widehat{\Omega}=\bigcup_{l} \widehat{\Omega}^{l} .
$$

A mapping $M^{l}$ is associated with level of refinement $l$ and is an approximation of $M$. Generally we take $M^{l}$ so that it maps a cell vertex from the partitioning $\widetilde{\Omega^{l}}$ to the same point in $\mathbb{R}^{2}$ as the original mapping $M$ does. Then in the sequence of mappings $\left\{M^{l}\right\}_{l \in \mathbb{Z}}$, $M^{l}$ approaches $M$ as $l \rightarrow \infty$. Hence, if $\overline{\Omega^{l}}=\overline{\widetilde{\Omega}}$, then $\overline{\Omega^{l}}$ is an approximation of $\bar{\Omega}$.

Since the partitioning of $\Omega$ on a level $l$ is regular quadrilateral, each cell on level $l$ can be denoted by $\Omega_{i, j}^{l} \subset \hat{\Omega}$. The set $I$ of indices present in the approximation is

$$
I=\left\{(i, j, l) \in \mathbb{Z}^{3} \mid \exists \Omega_{i, j}^{l} \subset \widehat{\Omega}\right\} .
$$

The grid on level $l$ is

$$
\Omega^{l}=\left\{\Omega_{i, j}^{l} \mid(i, j, l) \in I\right\} .
$$

By the regularity of the partitioning each cell on $\Omega^{l}$ has either none or only one neighbouring cell at each side, residing at the same level. A cell $\Omega_{i, j}^{l}$ is the northern neighbour of $\Omega_{i, j-1}^{l}$ and the eastern neighbour of $\Omega_{i-1, j}^{l}$, provided $(i, j-1, l),(i-1, j, l) \in I$. The boundary $\partial \Omega_{i, j}^{l} \subset \overline{\Omega_{i, j}^{l}}$ consists of the four cell faces of the cell, identified through their relative locations by $\partial \Omega_{i, j, k}^{l}, k \in D$, and $\partial \Omega_{i, j}^{l}=\bigcup_{k \in D} \partial \Omega_{i, j, k}^{l}$, where $D=\{N, E, S, W\}$.

Refinements of a cell $\Omega_{i, j}^{l}$ are the cells obtained by subdivision of the corresponding cell $\widetilde{\Omega}_{i, j}^{l}$ in the computational domain into $2 \times 2$ smaller cells of equal size. By applying the mapping $M^{l+1}$ to these refinements in the computational domain, we construct the refinements in the partitioning of the physical domain. Except for cells on the coarsest grid, each cell is one of the four descendants of a cell on the coarser grid. The coarsegrid cells on $\Omega^{l}$ and the fine-grid cells on $\Omega^{l+1}$ are coexistent (i.e. when cells appear on $\Omega^{l+1}$, the corresponding coarse-grid cells remain part of $\Omega^{l}$ ). A cell on the coarser grids is called parent and its descendants are called its kids. In this way all cells in the geometric structure belong to a quad-tree structure.

For the smallest integer coordinates on the coarsest grid, $\Omega^{0}$, without loss of generality we take $i=0$ and $j=0$. The integer coordinates of a cell on $\Omega^{l+1}$ are so that the kids of $\Omega_{i, j}^{l}$ are denoted by $\Omega_{2 i, 2 j}^{l+1}, \Omega_{2 i+1,2 j}^{l+1}, \Omega_{2 i, 2 j+1}^{l+1}$ and $\Omega_{2 i+1.2 j+1}^{l+1}$. A cell vertex in the physical domain is $P_{i, j}^{l}=M^{l}\left(\xi_{i, j}^{l}, \eta_{i, j}^{l}\right)$, where, without loss of generality, $\tilde{P}_{i, j}^{l}=\left(\xi_{i, j}^{l}, \eta_{i, j}^{l}\right)=\left(2^{-l} i, 2^{-l} j\right)$.

In this paper, functions. function spaces and subdomains defined for the computational domain are identified by a tilde on the same symbol used for the physical domain.

\subsection{The sequence of grids}

The geometric structure described so far, is used for multigrid computations on a locally refined grid. The grid $\Omega^{l_{b}}$ on some basic level $l_{b} \geq 0$ covers all $M^{l_{b}}(\widetilde{\Omega})$. The grids $\Omega^{l}$, 
$l>l_{b}$, are adaptively constructed during the computation, when it is decided that cells should be refined or refinements should be deleted, depending on the computed solution. At some stage in the computation. a sequence of grids $\left\{\Omega^{l}\right\}_{l=0, \ldots . L}$ has been generated, where $L$ is the highest level present. Thus, the cells on grid $\Omega^{l}, l>l_{b}$ typically do not cover all of the domain $\Omega$. Therefore. the grid $\Omega^{l}=\Omega_{f}^{l} \cup \Omega_{c}^{l}$ consists of a part $\Omega_{f}^{l}$ of which the cells have been refined (for which kids exist on level $l+1$ ) and a part $\Omega_{c}^{l}$, with cells that have not been refined (without kids). The set of all non-refined cells is called the composite grid $\Omega_{c}$, defined by.

$$
\Omega_{c}=\bigcup_{l=l_{b}}^{L} \Omega_{c}^{l} .
$$

Further, we define sets of indices associated with the different grids and parts of grids by $I^{l}=\left\{(i, j) \in \mathbb{Z}^{2} \mid(i . j, l) \in I\right\} . I_{f}^{l}=\left\{(i . j) \in I^{l} \mid \Omega_{i, j}^{l} \subset \Omega_{f}^{l}\right\}, I_{f}=\{(i, j, l) \in I \mid$ $\left.(i, j) \in I_{f}^{l}\right\}$. For practical purposes we also introduce $K$, defined by

$$
K(i, j)=\{(2 i .2 j),(2 i+1,2 j),(2 i .2 j+1),(2 i+1,2 j+1)\} .
$$

The boundary $\partial \Omega^{l}$ on level $l$ is $\partial \Omega^{l}=$ boundary of $\bigcup_{(i, j) \in I^{l}} \overline{\Omega_{i, j}^{l}}$. Following [14], the part of the boundary of the subdomain $\Omega^{l}$. which does neither coincide with a physical boundary nor with its discrete counterpart, is called a green boundary.

A grid is uniform if, in the computational domain, it covers all of $\tilde{\Omega}$ and if it is not refined anywhere; it is called locally uniform with respect to a discrete operator in a cell. if no green boundary is involved in the definition of the operator for that cell. A grid that contains locally non-uniform cells, is called a locally refined grid. Also a composite grid that consists of cells from more than one level is called locally refined.

\section{Finite volume discretisation on a locally refined grid}

\subsection{Grids and grid functions}

We see that the grid $\Omega^{l}$ on level $l$ consists of cells $\Omega_{i, j}^{l},(i, j) \in I^{l}$. A cell $\Omega_{i, j}^{l}$ in the physical domain is the result of $M^{l}\left(\tilde{\Omega}_{i, j}^{l}\right)$, where $M^{l}$ is an approximation of $M$ as described above. We assume that $M^{l}$ is continuous and piecewise affine on each cell face $\partial \widetilde{\Omega}_{i, j, k}^{l}$. Then the grid $\Omega^{l}$ in the physical space is a collection of disjoint quadrilaterals. In that situation, $M^{l}$ can be described as a vector of two continuous functions, both piecewise bilinear in each $\widetilde{\Omega}_{i, j}^{l}$. Furthermore we assume

$$
\left(P_{i, j}^{l}\right)^{T}=M^{l}\left(\tilde{P}_{i, j}^{l}\right)=M\left(\tilde{P}_{i, j}^{l}\right)
$$

i.e. $M^{l}$ is exact at the vertices $\tilde{P}_{i, j}^{l}$.

The boundary $\partial \Omega_{i, j}^{l}$ consists of the four faces $\partial \Omega_{i, j, k}^{l}, k=N, E, S, W$. A cell face $\partial \Omega_{i, j, k}^{l}$ has a length denoted by $s_{i, j, k}^{l}$. The area of a cell $\Omega_{i, j}^{l}$ is denoted by $A_{i, j}^{l}$.

If a function $u: \Omega^{l} \rightarrow \mathbb{R}^{d}$ is defined then also a function $\tilde{u}: \widetilde{\Omega}^{l} \rightarrow \mathbb{R}^{d}$ is defined through

$$
\tilde{u}(\xi, \eta)=u\left(x^{l}(\xi, \eta), y^{l}(\xi, \eta)\right),
$$


where $x^{l}$ and $y^{l}$ are given by

$$
\left(\begin{array}{l}
x^{l}(\xi . \eta) \\
y^{l}(\xi . \eta)
\end{array}\right)=M^{l}(\xi, \eta) .
$$

We denote the unknown vector function in our problem by $q$. The approximating function $q^{l}$ defined for the grid on level $l$. is a cell-wise constant function. The value of $q^{l}$ in a cell $\Omega_{i, j}^{l}$ is denoted by $q_{i, j}^{l}$, hence

$$
q_{i, j}^{l}=q^{l}\left(x^{\prime}, y^{\prime}\right) . \quad\left(x^{\prime}, y^{\prime}\right) \in \Omega_{i, j}^{l} \cap \Omega^{*} .
$$

The function value $q_{i, j}^{l}$ is called the state in cell $\Omega_{i . j}^{l}$. The space of all admissible state vectors is denoted by $X_{a} \subset \mathbb{R}^{d}$

\subsection{Restrictions}

In order to define the relations between the approximations on the different grids, it is appropriate to define a number of restrictions. The first is $\bar{R}^{l}$, the $L^{2}(\Omega)$-projection to the piecewise constant functions on $\Omega^{l}$. A projection closely related to this operator is $\bar{R}^{l, l+1}$, which restricts the piecewise constant functions on level $l$ to the refined part $\Omega_{f}^{l}$ of the grid. Next, we define the restriction $\bar{R}_{l+1}^{l}$, giving the piecewise constant function, which in each $\Omega_{i . j}^{l} \subset \Omega_{f}^{l}$ delivers the integral mean of the operand over its kids. I.e., if the collection of kids of $\Omega_{i, j}^{l}$ is

$$
\Sigma_{i, j}^{l}=\bigcup_{m \in K(i . j)} \Omega_{m}^{l+1},
$$

then the restriction $\bar{R}_{l+1}^{l}$ is defined by

$$
\left\{\bar{R}_{l+1}^{l} u\right\}_{i, j}^{l}=\frac{\int_{\Sigma_{i, j}^{l}} u d \Omega}{\int_{\Sigma_{i, j}^{l}} d \Omega} .
$$

Note that $\bar{R}^{l, l+1}=\bar{R}_{l+1}^{l}$, if the grid is obtained by .a piecewise bilinear mapping $M^{l}$ and $M^{l+1}=M^{l}$.

Another set of three restrictions (denoted without the overbar) gives the relations between vector functions in the r.h.s. space. The first restriction is the projection $R^{l}$, defined as the operator giving the cell-wise constant function consisting of the values if the integral of the operand on each cell on level $l$. The second restriction is the projection $R^{l, l+1}$, defined similarly as $\bar{R}^{l . l+1}$, i.e. $R^{l}$ restricted to the area covered by the fine grid $\Omega_{f}^{l}$. Finally we define a restriction $R_{l+1}^{l}$, which is related to $\bar{R}_{l+1}^{l}$ in (3.3) through the operators $A^{l}$ and $A^{l, l+1}$. which are defined as

$$
A^{l} u\left(x^{\prime}, y^{\prime}\right)=A_{i, j}^{l} u\left(x^{\prime}, y^{\prime}\right), \quad \forall\left(x^{\prime}, y^{\prime}\right) \in \Omega_{i, j}^{l},
$$

and

$$
A^{l . l+1} u\left(x^{\prime} \cdot y^{\prime}\right)=A^{l} u\left(x^{\prime}, y^{\prime}\right) . \quad \forall\left(x^{\prime}, y^{\prime}\right) \in \Omega_{i, j}^{l} \text { and }(i, j, l) \in I_{f} .
$$

With these definitions, we define the last restriction by

$$
R_{l+1}^{l}=A^{l, l+1} \bar{R}_{l+1}^{l}\left(A^{l+1}\right)^{-1} .
$$




\subsection{The system of discrete equations}

In this section we describe the system of equations obtained by the discretisation. We distinguish between equations obtained for cells on the composite grid and equations obtained for refined cells.

\section{Equations for a cell on a composite grid}

A discretisation of the set of conservation laws (1.1a) on a composite grid is obtained by considering the weak formulation of the problem: find $q$ from the solution space, satisfying the boundary conditions and so that for all $\Omega^{*} \subset \Omega$

$$
\int_{\Omega^{*}} \frac{\partial f(q(x, y))}{\partial x}+\frac{\partial g(q(x \cdot y))}{\partial y} d \Omega=\int_{\Omega^{*}} s d \Omega
$$

We assume that $q$ and $s$ are defined on $\widehat{\Omega}$. In case $\widehat{\Omega} \supset \Omega$, we assume that $q$ and $s$, defined on $\Omega$, can be extended to $\widehat{\Omega}$ in a sufficiently regular way. Then (3.5) is approximated by

$$
N(q)=r
$$

where $N$ and $r$ are functions defined on any $\Omega^{*} \subset \widehat{\Omega}$. For $\Omega_{i, j}^{l} \subset \widehat{\Omega}$ we define the restriction $R^{l} N$ by

$$
\left\{R^{l} N(q)\right\}_{i . j}^{l} \equiv \oint_{\partial \Omega_{i . j}^{l}} f(q) n_{x}+g(q) n_{y} d s
$$

where $n_{x}$ and $n_{y}$ are the components of the outward unit normal $n$ on the boundary $\partial \Omega_{i, j}^{l}$, in $x$ and $y$ direction respectively. The discretisation of the equations is obtained by requiring an approximation of (3.6) to hold for each cell on the composite grid. We first assume that for the discretisation the source term $s$ is exactly integrated. In our notation this implies

$$
r^{l}=R^{l} s \text {. }
$$

The mean value of the flux across the $k$ th cell face $\partial \Omega_{i, j, k}^{l} \subset \partial \Omega_{i, j}^{l}$ of cell $\Omega_{i, j}^{l}$ is

$$
f_{i, j, k}^{l}(q)=\frac{1}{s_{i, j, k}^{l}} \int_{\partial \Omega_{i, j, k}^{l}} f(q) n_{x}+g(q) n_{y} d s
$$

Hence, a solution of (3.6) exactly satisfies

$$
\left\{R^{l} N(q)\right\}_{i, j}^{l}=\sum_{k \in D} f_{i, j, k}^{l}(q) s_{i, j, k}^{l}=r_{i, j}^{l}, \quad \forall(i, j, l) \in I .
$$

Equations (3.8) are approximated by approximating the mean fluxes $f_{i, j, k}^{l}$ across each cell face $\partial \Omega_{i, j, k}^{l}$ by a numerical flux. denoted by $F_{i, j, k}^{l}$. In general this numerical flux depends on the functions $q^{m}, m=l_{b}, \ldots, l$. On a level $l$ the approximation of $(3.8)$ reads for all $\Omega_{i, j}^{l} \in \Omega_{c}^{l}$

$$
\sum_{k \in D} F_{i, j, k}^{l}\left(q^{l}: q^{l-1}, \ldots q^{l_{b}}\right) s_{i, j, k}^{l}=r_{i, j}^{l} .
$$

or in operator form

$$
N^{l}\left(q^{l} ; q^{l-1} \ldots \ldots q^{l_{b}}\right)=r^{l},
$$

where $N^{l}$ is defined by

$$
\left\{N^{l}\left(q^{l} ; q^{l-1}, \ldots, q^{l_{b}}\right)\right\}_{i, j}^{l}=\sum_{k \in D} F_{i, j, k}^{l}\left(q^{l} ; q^{l-1}, \ldots, q^{l_{b}}\right) s_{i, j, k}^{l} .
$$

Here, $q^{l-1}, \ldots, q^{l_{b}}$ act as parameters to $N^{l}$. These formulas define the discretisation on level $l$. 


\section{The numerical flux function}

The numerical Hux $F_{l, j, k}^{l}$ depends on the sequence $\left\{q^{m}\right\}_{m=l_{b}, \ldots, l}$. Usually we assume that the mumerical flux can be written as

$$
F_{l, j, k}^{l}\left(q^{l}: q^{l-1} \ldots \ldots q^{l_{b}}\right)=F\left(\left(q^{L}\right)_{i, j, k}^{l},\left(q^{R}\right)_{i, j, k}^{l}, n_{i, j, k}^{l}\right) .
$$

The arguments $\left(q^{L}\right)_{l, j, k}^{l}$ and $\left(q^{R}\right)_{i, j, k}^{l}$ denote estimates of the mean of $q$ along $\partial \Omega_{i, j, k}^{l}$, dependent on $\left\{q^{m}\right\}_{m=l_{h} \ldots . . l}$. with a bias to the left and right side of $\partial \Omega_{i . j, k}^{l}$, respectively. The entry $n_{i, j, k}^{l} \in E$ denotes the unit normal on $\partial \Omega_{i, j, k}^{l}$, pointing outward from $\Omega_{i, j}^{l}$, where $E \subset \mathbb{R}^{2}$ is the unit circle in $\mathbb{R}^{2}$. The function $F\left(q^{L}, q^{R} . n\right)$ is an approximation of the flux $f(q) n_{x}+g(q) n_{y}$. with $q^{L}$ and $q^{R}$ in the neighbourhood of $q$.

There are various wavs to define the states $q^{L}$ and $q^{R}$ and the numerical flux $F$. In fact. the choice of $F$ and the states $q^{L}$ and $q^{R}$ determine the discretisation method and its accuracy. The left and right states are usually obtained by piecewise polynomial reconstruction, using discrete data. i.e. using $\left\{q^{m}\right\}_{m=l_{b}, \ldots, l}$ (cf. Section 3.4).

For a hyperbolic set of conservation laws [10], we are interested in an upwind discretisation. which may be obtained by taking for $F$ an (approximate) Riemann solver. The best-known approximate Riemann solvers are introduced in $[13,22,12]$.

\section{Equations for a refined cell}

Discrete equations (3.9) are approximations of the conservation equation (3.5) for each cell that has not been refined. The left and right state for the computation of a numerical flux depend on the states in neighbouring cells. possibly on different levels. By definition, for a locally non-uniform grid cell. the left or right state for at least one cell face depends on coarse-grid states.

The set of equations obtained by applying the discretisation as described at the beginning of this section are under-determined for a locally refined grid. If a neighbouring cell has been refined (has kids), that neighbour is not part of the composite grid, and no equation like (3.9) has been defined. Additional equations, however, are obtained by

$$
q_{i, j}^{l}=\left\{\bar{R}_{l+1}^{l} q^{l+1}\right\}_{i, j}^{l}, \quad \forall(i, j, l) \in I_{f} .
$$

We use the equations (3.9) together with (3.10) to define discretisations on a locally refined (i.e. composite) grid.

\subsection{Left and right states}

Here we describe the computation of $\left(q^{L}\right)_{i, j, k}^{l}$ and $\left(q^{R}\right)_{i, j, k}^{l}$, the left and right state used in the numerical flux function. We consider first and second-order accurate discretisations. both for a locally uniform and a locally non-uniform situation. We use the concept of reconstruction of piecewise $C^{\infty}$-functions from the cell-wise constant data. $q_{i, j}^{l}$, associated with each cell. This idea was introduced in [21] and [24] for one-dimensional convection and extended and applied in [1] and [2] for unstructured grids in two spatial dimensions. Contrary to this work, we do not reconstruct a single, unique (vector) function in each cell, but we take care that -in a locally non-uniform grid situation- the computation of the left and right state is so that the resulting scheme is consistent of the required order (at least in some weak sense, see Section 4). This is done by making a different reconstruction for each side of each cell face. 


\section{Locally uniform composite grids}

The computation of the first-order as well as of the second-order consistent discretisation depends on the mean states. On a locally uniform grid, first-order consistency is obtained by applying an $\mathcal{O}\left(h_{l}\right)$ accurate reconstruction. Consider for example the eastern cell face $\partial \Omega_{i, j, E}^{l}$ of a cell $\Omega_{i, j}^{l}$ on a locally uniform composite grid, where $(i+1, j) \in I^{l}$. For this situation we take for the states, as usual in first-order Godunov-type schemes, [4, 8, 18],

$$
\begin{aligned}
& \left(q^{L}\right)_{i, j, E}^{l}=q_{i, j}^{l}, \\
& \left(q^{R}\right)_{i, j, E}^{l}=q_{i+1 . j}^{l} .
\end{aligned}
$$

For second-order consistency on a locally uniform grid, the states are based on $\mathcal{O}\left(h_{l}^{2}\right)$ accurate reconstructions of the state functions. This reconstruction can be done with a limiter to suppress spurious wiggling of the solution (as proposed e.g. in $[15,19]$ and applied in [9]), or without a limiter, like the $i$-schemes [23] (as e.g. in [7, 16] and [9]). Again, for the eastern cell face of a cell $\Omega_{i, j}^{l}$ on a locally uniform grid, where $(i-1, j),(i+$ $1, j),(i+2, j) \in I^{l}$, the limiter and $\kappa$-schemes are given by

$$
\begin{aligned}
& \left(q^{L}\right)_{i, j, E}^{l}=C\left(q_{i-1, j}^{l}, q_{i, j}^{l}, q_{i+1, j}^{l}\right), \\
& \left(q^{R}\right)_{i, j, E}^{l}=C\left(q_{i+2, j}^{l}, q_{i+1, j}^{l}, q_{i, j}^{l}\right),
\end{aligned}
$$

where, $C: X_{a} \times X_{a} \times X_{a} \rightarrow X_{a}$ describes the $\kappa$-scheme or the limiter scheme. Notice that the $\kappa$-schemes are recovered by applying certain linear 'limiter' functions. However a $\kappa$-scheme does not necessarily satisfy monotonicity conditions, see [17].

\section{Locally non-uniform grids}

On a locally refined grid, one or more of the mean states in (3.11) or (3.12) are not available, because the cells with which the states should be associated. do not exist. For this, we introduce the concept of virtual cell and associated virtual state.

With each integer coordinate pair $\left(2^{n} i+r, 2^{n} j+s\right) \notin I^{l+n}, 0 \leq r, s<2^{n}, n \geq 1$ and $(i, j) \in I^{l}, l_{b} \leq l \leq L-n$, we associate the virtual cell $\tilde{\omega}_{2^{n} i+r, 2^{n} j+s}^{l+n} \subset \tilde{\Omega}$, given by

$$
\begin{aligned}
\tilde{\omega}_{2^{n} i+r, 2^{n} j+s}^{l+n}= & 2^{-(l+n)}\left(2^{n} i+r, 2^{n} i+r+1\right) \\
& \times 2^{-(l+n)}\left(2^{n} j+s, 2^{n} j+s+1\right) .
\end{aligned}
$$

In the physical space the virtual cell $\omega_{2^{n} i+r, 2^{n} j+s}^{l+n} \subset \widehat{\Omega}$ is defined as

$$
\omega_{2^{n} i+r, 2^{n} j+s}^{l+n}=M^{l+n}\left(\widetilde{\omega}_{2^{n} i+r, 2^{n} j+s}^{l+n}\right) .
$$

Note that $\omega_{r, s}^{l+n}$ is exactly $\Omega_{r, s}^{l+n}$, if the grid would be sufficiently refined.

With the virtual cell $\omega_{i, j}^{l}$ we associate a virtual state $v_{i, j}^{l} \in X_{a}$, which can be interpreted as an approximation of the mean of the state vector function on $\omega_{i, j}^{l}$. In general a virtual state $v_{i, j}^{l}$ depends on $\left\{q^{m}\right\}_{m=l_{b}, \ldots, l}$.

Virtual cells and virtual states are used for the discretisation in the neighbourhood of green boundaries. To a large extent the virtual states determine the accuracy of the algebraic equations associated with the locally non-uniform grid.

The concept of virtual states allows us to compute left and right states in a locally non-uniform grid situation, in a way similar to (3.11) and (3.12). The requirements 
to be satisfied for the proper computation of virtual states, are discussed in Section 4 . Regardless of the way how the virtual states are computed, for first-order consistency we take for the eastern cell face of $\Omega_{i, j}^{l}$. similarly to $(3.11)$, and $(i+1 . j) \notin I^{l}$

$$
\begin{aligned}
& \left(q^{L}\right)_{i, j, E}^{l}=q_{i, j}^{l}, \\
& \left(q^{R}\right)_{i, j, E}^{l}=v_{i+1, j}^{l} .
\end{aligned}
$$

Similar to (3.12), we take for second-order consistency, if $(i+1, j),(i+2, j) \notin I^{l}$

$$
\begin{aligned}
& \left(q^{L}\right)_{i, j, E}^{l}=C\left(q_{i-1, j}^{l}, q_{i, j}^{l}, v_{i+1, j}^{l}\right), \\
& \left(q^{R}\right)_{i, j, E}^{l}=C\left(v_{i+2, j}^{l}, v_{i+1, j}^{l}, q_{i, j}^{l}\right) .
\end{aligned}
$$

and if $(i+1, j) \in I^{l}$. but $(i+2, j) \notin I^{l}$

$$
\begin{aligned}
& \left(q^{L}\right)_{i, j, E}^{l}=C\left(q_{i-1, j}^{l}, q_{i, j}^{l}, q_{i+1, j}^{l}\right), \\
& \left(q^{R}\right)_{i, j, E}^{l}=C\left(v_{i+2, j}^{l}, q_{i+1, j}^{l}, q_{i, j}^{l}\right),
\end{aligned}
$$

Formulae similar to (3.14)-(3.16) are used for the cell faces, $\partial \Omega_{i, j, k}^{l}, k=N, S . W$.

\section{Error analysis of the discretisation}

In this section we study the local discretisation error and the consistency of the discretisation described in Section 3. In the discretisation we distinguish three approximations, each of which have a contribution to the local discretisation error. These contribution are:

- approximation of the mapping from the computational space into the physical space; in equations this error is denoted by $\tau_{m}^{l}(q)$;

- approximation of the mean flux on a cell face by the flux evaluated at the mean state along the cell face (quadrature rule); in equations this error is denoted by $\tau_{q}^{l}(q)$;

- approximation of the mean state on a cell face by biased reconstructions; in equations this error is denoted by $\tau_{r}^{l}(q)$.

Often the approximation of $M$ by $M^{l}$ is not essential, since the change from $M$ to $M^{l}$, merely changes the partitioning in the physical domain slightly, without affecting the accuracy of the resulting set of algebraic equations. However, it does affect the approximation of the domain of definition of the problem. Hence, for the interior cells of $\tilde{\Omega}$ we can assume $M=M^{l}=M^{l_{0}}$, for some constant $l_{0}$ and $l_{0} \leq l \leq L$, without affecting the accuracy. At the domain-boundary, $\partial \widetilde{\Omega}$, the error of this approximation $M^{l}$ can be important. Here, in general we do not have $M=M^{l}=M^{l_{0}}$. because it results in an approximation of the boundary, independent of the level $l$. Hence, with increasing levels of refinement, the geometry of the discrete problem would not converge to the geometry of the continuous problem. A more important reason to study this approximation is related to a-posteriori estimation of the local discretisation error and the application of $\tau$-extrapolation. This uses the so-called relative local discretisation errors of two consecutive levels of refinement (cf. $[3,20])$. 
The two errors $\tau_{q}^{l}(q)$ and $\tau_{r}^{l}(q)$ together make up the local truncation error. For the fluxes approximated with the projection of the true solution. i.e. with $q=\bar{q}$, the exact solution of the problem. they form the error in the system of algebraic equations. for a given partitioning.

The analysis presented in this section leads to recquirements to be imposed on the reconstruction of cell-wise smooth functions from the cell-wise constant numerical data. These requirements. depend on the goal set out to be reached in a particular discretisation. We distinguish between the goals (i) obtain a given order of consistency and (ii) obtain a given order of discrete convergence. This distinction is made. since a certain order of the local discretisation error for all equations may not be essential to obtain a given order of discrete convergence. Assume a total number of equations of $\mathcal{O}\left(h^{-2}\right)$. An $\mathcal{O}\left(h^{-1}\right)$ number of equations with low-order accuracy may not affect the rate of discrete convergence, not even in supremum norm. To study this in detail. we have to redefine the notion of consistency for a non-uniform mesh. In fact, we define a slightly weaker form of consistency. This weaker form is the discrete $L_{1}$-norm of the local truncation error for a collection of discrete equations. The requirements for consistency both in the weak and in the usual sense are studied.

\subsection{Approximation of the mapping}

To make an a-posteriori estimation of the local discretisation error, with sufficient accuracy, we first study the consequences of approximating $M$ by $M^{l}$. Actually, we are only interested in the relation between the mappings for two consecutive levels of refinement, since we want to study the use of two consecutive levels of refinement in the estimation of the local discretisation error. The relation between the mappings of two consecutive levels can be established through their relation to $M$. This relation also allows us study the accuracy of the restriction to the coarse grid (cf. Section 4.2).

We consider a surjective mapping $M: \overline{\widetilde{\Omega}} \rightarrow \bar{\Omega}$, also injective on $\tilde{\Omega}$. We also consider its continuous. piecewise bilinear approximation associated with level of refinement $l$, $M^{l}: \overline{\widetilde{\Omega}} \rightarrow \bar{\Omega}^{l}$, which is exact in the vertices $\tilde{P}_{i, j, k}^{l}$. To simplify notations, in the present local analysis, we drop the indices which are constant throughout this part of the analysis. We consider a cell on level of refinement $l$. In the computational space. the corresponding cell is denoted by $\widetilde{\Omega}$. Its images in $\widehat{\Omega}$ are

$$
\begin{aligned}
\Omega & =M(\tilde{\Omega}), \\
\Omega^{l} & =M^{l}(\tilde{\Omega}),
\end{aligned}
$$

where we drop the subscripts. We use a local Cartesian coordinate system $(\xi, \eta)$ in the computational space, with its origin in the center of $\widetilde{\Omega}$. Similar to (3.2), we use the Cartesian coordinates in the physical space as obtained by $M$

$$
\left(\begin{array}{l}
x(\xi, \eta) \\
y(\xi, \eta)
\end{array}\right)=M(\xi, \eta)
$$

where the origin in the physical space is

$$
(0,0)^{T}=M(0,0) .
$$

We assume $\tilde{\Omega}$ being a square with edges $h_{l}=2 h, \tilde{\Omega}=(-h, h)^{2}$. The area in the computational space is $4 h^{2}$. In the physical space the area is denoted by $A$ for $\Omega$ and $A^{l}$ for $\Omega^{l}$. 
The Jacobians of $M$ and $M^{l}$ are denoted by $J$ and $J^{l}$ respectively:

$$
\begin{aligned}
J(\xi, \eta) & =x_{\xi} y_{\eta}-x_{\eta} y_{\xi}, \\
J^{l}(\xi, \eta) & =x_{\xi}^{l} y_{\eta}^{l}-x_{\eta}^{l} y_{\xi}^{l},
\end{aligned}
$$

where the subscripts denote differentiation with respect to $\xi$ and $\eta$ respectively. Further, we assume that $M$ is sufficiently smooth and for $(\xi, \eta) \in \overline{\widetilde{\Omega}}$ it can be written

$$
\begin{aligned}
M= & m_{0}+m_{1} \xi+m_{2} \eta+m_{3} \xi^{2}+m_{4} \xi \eta+m_{5} \eta^{2} \\
& +m_{6} \xi^{3}+m_{7} \xi^{2} \eta+m_{8} \xi \eta^{2}+m_{9} \eta^{3}+\ldots
\end{aligned}
$$

Note that by $(4.1) m_{0}=(0,0)^{T}$. The mapping $M^{l}$ is piecewise bilinear:

$$
M^{l}=m_{0}^{l}+m_{1}^{l} \xi+m_{2}^{l} \eta+m_{4}^{l} \xi \eta .
$$

We define $\left(x_{i}, y_{i}\right)^{T},\left(x_{i}^{l}, y_{i}^{l}\right)^{T}$ by

$$
\begin{array}{ll}
m_{i}=\left(x_{i}, y_{i}\right)^{T}, & i=0.1,2, \ldots, \\
m_{i}^{l}=\left(x_{i}^{l}, y_{i}^{l}\right)^{T}, & i=0.1,2,4 .
\end{array}
$$

From the exactness of $M^{l}$ at the cell vertices. given by (3.1), we can express $m_{i}^{l}, i=$ $0,1,2,4$ in terms of $m_{i}, i=0,1, \ldots$. This gives

$$
\begin{aligned}
& m_{0}^{l}=m_{0}+m_{3} h^{2}+m_{5} h^{2}+\mathcal{O}\left(h^{4}\right), \\
& m_{1}^{l}=m_{1}+m_{6} h^{2}+m_{8} h^{2}+\mathcal{O}\left(h^{4}\right), \\
& m_{2}^{l}=m_{2}+m_{7} h^{2}+m_{9} h^{2}+\mathcal{O}\left(h^{4}\right), \\
& m_{4}^{l}=m_{4}+\mathcal{O}\left(h^{4}\right) .
\end{aligned}
$$

As a result, we have $M^{l}$ expressed in terms of $M$, for $|\xi|,|\eta| \leq h$, and

$$
M^{l}-M=m_{3}\left(h^{2}-\xi^{2}\right)+m_{5}\left(h^{2}-\eta^{2}\right)+\mathcal{O}\left(h^{3}\right) .
$$

We are now interested in the difference between the weak form (3.5), for $\Omega=M(\widetilde{\Omega})$ and $\Omega^{l}=M^{l}(\tilde{\Omega})$, divided by the respective areas $A$ and $A^{l}$. This error is denoted by $\tau_{m}(q)$.

Let a sufficiently smooth, integrable function $w: \Omega \cup \Omega^{l} \rightarrow \mathbb{R}^{d}$ be defined, and let its Taylor series expansion around the origin $(x, y)=(0,0)$ be given by

$$
w(x, y)=w_{0}+w_{1} x+w_{2} y+w_{3} x^{2}+\ldots
$$

For the error of the weak form on $\Omega^{l}$ with respect to the weak form on $\Omega$, it suffices to consider the difference

$$
t(w) \equiv \frac{1}{A^{l}} \int_{\Omega^{l}} w^{\prime} d \Omega-\frac{1}{A} \int_{\Omega} w d \Omega .
$$

for a sufficiently smooth and integrable function $w$. For $M$ and $M^{l}$, assuming from this point that $J . J^{l}>0$. this is equal to

$$
t(w)=\frac{1}{A^{l}} \int_{\tilde{\Omega}} w^{l} J^{l} d \tilde{\Omega}-\frac{1}{A} \int_{\tilde{\Omega}} w J d \tilde{\Omega},
$$


where $w^{l}=w\left(x^{l} \cdot y^{l}\right)$. With

$$
\begin{aligned}
& \delta_{w}=w^{l}-w, \\
& \delta_{J}=J^{l}-J
\end{aligned}
$$

the integrand $w^{l} J^{l}$ can be written as

$$
w^{l} J^{l}=w J+\delta_{w} J+w \delta_{J}+\delta_{w} \delta_{J}
$$

An expression for $\delta_{w}$ in terms of $m_{i}$ and $w_{i}$ can be obtained by using (4.5) for $\left(x^{l}, y^{l}\right)$ and $(x . y)$, and by subtraction and substitution of (4.4). From this exercise it appears that $\delta_{w}=\mathcal{O}\left(h^{2}\right)$. Multiplication with $J$ and integration over $\widetilde{\Omega}$ yields

$$
\int_{\tilde{\Omega}} \delta_{w} J d \tilde{\Omega}=\frac{8}{3} J_{0} h^{4}\left\{w_{1}\left(x_{3}+x_{5}\right)+w_{2}\left(y_{3}+y_{5}\right)\right\}+\mathcal{O}\left(h^{5}\right),
$$

where $J_{0}$ is $J(0,0)$.

Similarly, based on (4.4), we can also find an expression for $\delta_{J}$. It appears that generally $\delta_{J}=\mathcal{O}(h)$, with the $\mathcal{O}(h)$ terms linear in $\xi$ or $\eta$. A straightforward calculation yields for the integral of $w \delta_{J}$

$$
\begin{aligned}
& \int_{\tilde{\Omega}} w \delta_{J} d \tilde{\Omega}= \\
& \frac{8}{3} h^{4}\left\{w_{0}\left(x_{1} y_{7}-x_{7} y_{1}+x_{8} y_{2}-x_{2} y_{8}-x_{3} y_{4}+x_{4} y_{3}-x_{4} y_{5}+x_{5} y_{4}\right)\right. \\
& \quad+w_{1}\left(-x_{1} x_{3} y_{2}+x_{1} x_{2} y_{3}-x_{1} x_{2} y_{5}+x_{2} y_{5} y_{1}\right) \\
& \left.\quad+w_{2}\left(-x_{3} y_{1} y_{2}+x_{2} y_{1} y_{3}-x_{1} y_{2} y_{5}+x_{5} y_{1} y_{2}\right)\right\}+\mathcal{O}\left(h^{5}\right) .
\end{aligned}
$$

The term $\delta_{w} \delta_{J}$ gives only an $\mathcal{O}\left(h^{5}\right)$ contribution to the integral over $\tilde{\Omega}$.

By (4.7) and (4.8) we can define $C(w)$ as the coefficient in the first term of an asymptotic expansion, given by the sum of (4.7) and (4.8), i.e.

$$
\int_{\tilde{\Omega}}\left(w^{l} J^{l}-w J\right) d \tilde{\Omega}=h^{4} C(w)+\mathcal{O}\left(h^{5}\right),
$$

with $C$ independent of $h$. Now we can write

$$
A^{l}-A=h^{4} C(1)+\mathcal{O}\left(h^{5}\right) .
$$

For the area $A$ we have

$$
A=4 h^{2} J_{0}+\mathcal{O}\left(h^{4}\right),
$$

combination of (4.9) and (4.10) yields after some manipulation

$$
\frac{1}{A^{l}}-\frac{1}{A}=-\frac{C(1)}{16 J_{0}^{2}}+\mathcal{O}(h) .
$$

Using this, it can be easily shown that the difference $t(w)$ satisfies

$$
t(w)=\frac{h^{2}}{4 J_{0}}\left(C(w)-w_{0} C(1)\right)+\mathcal{O}\left(h^{3}\right) .
$$

With

$$
\tau_{m}^{l}(q)=t\left(\frac{\partial f(q)}{\partial x}+\frac{\partial g(q)}{\partial y}\right)
$$


we establish the asymptotic relation between the approximation of the weak form on two consecutive levels of refinement, caused by the approximation of the grid geometry. Although the difference between the partitioning obtained with $M$ and the partitioning obtained with $M^{l}$ itself is not essential for the convergence of the local discretisation error, we use the result (4.11) when we show that an a-posteriori error estimation is sufficiently accurate. Further, we use (4.11) to show that on a composite grid, the equations for a refined cell (3.10), in general give at best second-order accuracy on the coarse grid. Assume for (4.11) that $w$ is the differential operator, applied to the solution of the continuous problem. According to the result in (4.11), in general the discrete equations derived for boundary cells are at most second-order accurate, because (in general) we cannot use $M=M^{l}=M^{l_{0}}, l_{0}$ a constant, $l_{0} \leq l \leq L$, in cells along the boundary of the domain. A piecewise bilinear mapping $M=M^{l}$ would yield $\tau_{m}^{l}(q)=0$.

\subsection{Accuracy of the coarse-grid restriction}

We consider the restriction $\bar{R}_{l+1}^{l}$, as defined by (3.3). We study the difference between the restrictions $\bar{R}_{l+1}^{l} \bar{R}^{l+1} q$ (cf. (3.3)) and $\bar{R}^{l, l+1} q$. In the computation of the virtual states we use as the coarse-grid discrete function on $\Omega_{f}^{l}$, the restricted function $q_{i, j}^{l}=\left\{\bar{R}_{l+1}^{l} q^{l+1}\right\}_{i, j}^{l}$, $(i, j) \in I_{f}^{l}$, where $q^{l+1}=\bar{R}^{l+1} q$, while we assume that $q_{i, j}^{l}$ is the mean of $q$ on $\Omega_{i, j}^{l}$, given by $\bar{R}^{l, l+1} q$. The error we make with this assumption is studied here.

In our analysis we again drop the unnecessary indices. We consider a cell $\Omega^{l}$ on level $l$, which has refinements on level $l+1$. A kid of $\Omega^{l}$ is identified by the subscript $m \in K$, where $K$ is the set of indices (cf. (2.2)), associated with the cell $\Omega^{l}$. For level $l$ we have the approximation $M^{l}$ of $M$ and for level $l+1$ we have $M^{l+1}$. Similar to the notations in the previous subsection, we use the superscripts in our notations to distinguish between variables for $\Omega=M(\tilde{\Omega})$ (e.g. $A$, no superscript) and $\Omega^{l}=M^{l}(\tilde{\Omega})$ (e.g. $A^{l}$ ). Hence, e.g. $A_{m}^{l+1}, m \in K$, denotes the area of the $m$ th kid $M^{l+1}\left(\widetilde{\Omega}_{m}^{l+1}\right)$.

Consider $\bar{R}_{l+1}^{l} q^{l+1}$, where $q^{l+1}=\bar{R}^{l+1} q$. By definition of $\bar{R}_{l+1}^{l}$ we have

$$
\bar{R}_{l+1}^{l} \bar{R}^{l+1} q=\frac{\sum_{m \in K} A_{m}^{l+1} q_{m}^{l+1}}{\sum_{m \in K} A_{m}^{l+1}}
$$

With the results from the previous subsection we find

$$
\frac{1}{\sum_{m \in K} A_{m}^{l+1}}=\frac{1}{A}-\frac{h_{l+1}^{4} C(1)}{4 A^{2}}+\mathcal{O}\left(h_{l+1}\right) .
$$

For the numerator in (4.12) we find

$$
\sum_{m \in K^{K}} A_{m}^{l+1} q_{m}^{l+1}=\int_{\tilde{\Omega}} q J d \tilde{\Omega}+\frac{1}{4} h_{l+1}^{4} C(q)+\mathcal{O}\left(h_{l+1}^{5}\right) .
$$

Multiplication of (4.13) and (4.14) gives for (4.12):

$$
\bar{R}^{l, l+1} \bar{R}^{l+1} q=\frac{1}{A} \int_{\widetilde{\Omega}} q J d \widetilde{\Omega}+\frac{h_{l+1}^{2}}{16 J_{0}}\left(C(q)-q_{0} C(1)\right)+\mathcal{O}\left(h_{l+1}^{3}\right) .
$$

With (4.6) and (4.11) we obtain

$$
\bar{R}^{l} q=\frac{1}{A} \int_{\tilde{\Omega}} q J d \tilde{\Omega}+\frac{h_{l+1}^{2}}{4 J_{0}}\left(C(q)-q_{0} C(1)\right)+\mathcal{O}\left(h_{l}^{3}\right) .
$$


Hence, the difference between the two restrictions is

$$
\bar{R}^{l, l+1} q-\bar{R}_{l+1}^{l} \bar{R}^{l+1} q=\frac{3}{16} \frac{h_{l+1}^{2}}{J_{0}}\left(C(q)-q_{0} C(1)\right)+\mathcal{O}\left(h_{l}^{3}\right) .
$$

\subsection{Consistency and weak consistency}

Now we consider the approximation of the weak form on a given partitioning by the set of discrete equations. We assume that $M=M^{l}=M^{l_{0}}$, where $l_{0}<l$. With (2.1) this implies $\Omega=M^{l}(\tilde{\Omega})$. This in turn implies that we do not consider the differences between the discretisation on different levels, due to the differences in mappings from the computational into the physical space, on each level.

\section{Local discretisation error and consistency}

We use the notation as defined in Section 2 and 3. Let $N(q)=r$ denote the continuous equations (3.5). We denote the solution of this continuous problem by $\bar{q}$ :

$$
N(\bar{q})=r .
$$

Let the non-linear operator $N$ be approximated by the discrete finite-volume operator $N^{l}$, on a level of refinement $l \in\{0 \ldots, L\}$, of a sufficiently smooth grid, consisting of quadrilaterals. Further. let projections $R^{l}$ and $\bar{R}^{l}$ be defined properly, for example by the definitions in Section 3.2, and related to each other through $A^{l}$, as in (3.4). Assume that the right-hand side of (1.1a) is exactly integrated, as denoted by (3.7). The local discretisation error for $N^{l}\left(q^{l} ; q^{l-1}\right)=r^{l}$ is $\tau^{l}(\bar{q})$, where $\tau^{l}$ is given by

$$
\tau^{l}(q)=\left(A^{l}\right)^{-1}\left(N^{l}\left(\bar{R}^{l} q ; \bar{R}^{l-1} q\right)-R^{l} N(q)\right) .
$$

We define $\tau_{i, j}^{l}(q) \equiv\left\{\tau^{l}(q)\right\}_{i, j}^{l}$. The discretisation $N^{l}$ is called an approximation to $N$ of order of consistency $p$, if for all $(i, j, l) \in I$

$$
\tau_{i, j}^{l}(\bar{q})=\mathcal{O}\left(h_{l}^{p}\right),
$$

for $h_{l} \rightarrow 0$. Notice that $h_{l} \rightarrow 0$, if $l \rightarrow \infty$.

\section{Weak consistency}

We also introduce a weak form of consistency, related to the above mentioned consistency in the usual sense. The new definition of consistency is weaker than the usual definition, because it considers the collective behaviour of the local discretisation error for a set of equations, rather than the behaviour of each equation separately. Consider a partitioning of the domain $\Omega$, obtained by refining $n$ times a previously obtained locally refined composite grid. Let a discretisation as described in Section 3 be defined for this new system. Then the collective local truncation error, $T_{n}^{l}(q)$, for an $n$ times completely refined system is defined by

$$
T_{n}^{l}(q)=A^{-1} R_{l+1}^{l} \ldots R_{l+n}^{l+n-1}\left(N^{l+n}\left(\bar{R}^{l+n} q\right)-R^{l+n} N(q)\right) .
$$

In absolute value, this is the discrete $L_{1}$-norm of the local truncation error for the set of equations for all descendants of each cell $\Omega_{i, j}^{l}$. Note that

$$
T_{0}^{l}(q)=\tau^{l}(q) .
$$


A discretisation $V^{i}$ is called weakly consistent of order $p$ if

$$
\left\{T_{n}^{l}(\bar{q})\right\}_{i, j}^{l}=\mathcal{O}\left(h_{l+n}^{p}\right) . \quad \forall(i, j, l) \in I .
$$

for $n \rightarrow \infty$.

\subsection{Analysis of local truncation error}

In this section we study the local truncation error of the discretisation introduced in Section 3. We use the same assumptions as formulated at the beginning of Section 4.3. From the analysis we obtain requirements for the computation of the virtual states, both for a consistent and weakly consistent discretisation.

As described at the beginning of Section 4. the local discretisation error consists of two contributions. The first contribution. denoted by $\tau_{q}^{l}(q)$, is a result of the quadrature rule that is used to approximate the mean value along a cell face, of the flux across that cell face. This mean value is approximated by the flux evaluated at the mean value of the state function along that face. Further. the mean value of the state function $q$ along a cell face is approximated by reconstruction of piecewise polynomial functions from cell-wise constant functions which represents the average of $q$ in each cell. The reconstruction is done twice for each cell face, i.e. for each of the two sides. with a bias in both opposite directions. These reconstructed approximations of the mean value along a cell face is then used in an approximate Riemann solver. If $q$ is a solution of the continuous equation (1.1a), this procedure gives a contribution to the local discretisation error, denoted by $\tau_{r}^{l}(q)$

\section{Quadrature rule}

Similar as before. we drop the indices which are superfluous here. As noted, the mapping $M$ is assumed to be bilinear on $\tilde{\Omega}$. It is given by

$$
M(\xi, \eta)=m_{0}+m_{1} \xi+m_{2} \eta+m_{4} \xi \eta,
$$

where $m_{0}, m_{1}, m_{2}$ and $m_{4}$ are defined by (4.3). Note that the cell-wise constant parameters $m_{2}$ are fully determined by the coordinates of the vertices of that cell. The area of cell $\Omega$ is

$$
A=\int_{\Omega} d \Omega=\int_{\tilde{\Omega}} J d \tilde{\Omega}=4 h^{2} J_{0}
$$

where $J_{0}=x_{1} y_{2}-x_{2} y_{1}>0$, the Jacobian of $M$ at the origin.

We denote the mean value of the flux across the $k$ th cell face by $f_{k}$ and since $M$ is linear at $\partial \tilde{\Omega}_{k}$, we have

$$
\begin{aligned}
f_{k}(q) & =\frac{1}{s_{k}} \int_{\partial \Omega_{k}} f(q) n_{x}+g(q) n_{y} d s \\
& =\frac{1}{2 h} \int_{\partial \tilde{\Omega}_{k}} f(q) n_{x}+g(q) n_{y} d \tilde{s} .
\end{aligned}
$$

The mean of $q(x, y)$ along $\partial \Omega_{k}$ is denoted by

$$
q_{k}=\frac{1}{s_{k}} \int_{\partial \Omega_{k}} q d s .
$$

The unit normal along a cell face is constant, and we use the notation

$$
\left(n_{x}\right)_{k}=\left.n_{x}\right|_{\partial \Omega_{k}}, \quad\left(n_{y}\right)_{k}=\left.n_{y}\right|_{\partial \Omega_{k}} .
$$


By $q^{*}$ and $q_{k}^{*}$ we denote

$$
\begin{aligned}
& q^{*}=\left(q, n_{x}, n_{y}\right)^{T}, \\
& q_{k}^{*}=\left(q_{k},\left(n_{x}\right)_{k},\left(n_{y}\right)_{k}\right)^{T},
\end{aligned}
$$

and by $f^{*}$ we denote the flux in the direction of $n=\left(n_{x}, n_{y}\right)^{T}$, given by

$$
f^{*}\left(q^{*}\right)=f(q) n_{x}+g(q) n_{y} .
$$

The contribution $\tau_{q}(q)$ of the quadrature rule to the local truncation error is given by

$$
\tau_{q}(q)=\frac{1}{A} \sum_{k \in D}\left(f^{*}\left(q_{k}^{*}\right)-f_{k}(q)\right) s_{k} .
$$

An expansion of $f^{*}\left(q^{*}\right)$ around $q_{k}^{*}$ yields

$$
\begin{aligned}
f^{*}\left(q^{*}\right)=f^{*}\left(q_{k}^{*}\right) & +\left.\frac{\partial f^{*}}{\partial q^{* \alpha}}\right|_{q_{k}^{*}}\left(q^{*}-q_{k}^{*}\right)^{\alpha} \\
& +\left.\frac{1}{2} \frac{\partial^{2} f^{*}}{\partial q^{* \alpha} \partial q^{*}}\right|_{q_{k}^{*}}\left(q^{*}-q_{k}^{*}\right)^{\alpha}\left(q^{*}-q_{k}^{*}\right)^{\beta}+\ldots,
\end{aligned}
$$

where the superscripts denote the components of $q^{*}$ and $q_{k}^{*}$, and the summation convention is used for $\alpha, \beta=1, \ldots, d+2$. We assume that the function $q(x(\xi, \eta), y(\xi, \eta))$ can be written as a Taylor series expansion around the origin of the local Cartesian coordinate system in the computational space. Now some computation, the details of which are found in [20] shows that the contribution of the quadrature rule to the local discretisation error is $\mathcal{O}\left(h^{2}\right)$.

\section{Reconstruction}

Here we study the role of the reconstruction step, in which the left and right states are computed. This yields requirements which should be satisfied by the reconstruction, in order to obtain a consistent or a weakly consistent discretisation. In the previous subsections we found that the local truncation error is limited to second-order by the choice of the mapping and by the choice of quadrature rule. Hence, we are interested in first and second-order consistency only.

In our notations we again drop indices if convenient. As we are interested in a local discretisation error, which is $\mathcal{O}\left(h^{p}\right), p=1.2$. we consider the equation for a cell $\Omega$ on some level in the geometric structure. We assume that the solution of the problem is sufficiently smooth and that the numerical flux function is sufficiently differentiable.

The outward pointing unit normals on $\partial \Omega_{k}$ are given by

$$
\begin{array}{ll}
n_{E}=\left.\left(\begin{array}{l}
y_{\eta} \\
-x_{\eta}
\end{array}\right) \frac{1}{\sqrt{x_{\eta}^{2}+y_{\eta}^{2}}}\right|_{\partial \Omega_{E}}, \quad n_{S}=\left.\left(\begin{array}{l}
y_{\xi} \\
-x_{\xi}
\end{array}\right) \frac{1}{\sqrt{x_{\xi}^{2}+y_{\xi}^{2}}}\right|_{\partial \Omega_{S}}, \\
n_{W}=\left.\left(\begin{array}{l}
-y_{\eta} \\
x_{\eta}
\end{array}\right) \frac{1}{\sqrt{x_{\eta}^{2}+y_{\eta}^{2}}}\right|_{\partial \Omega_{W}} . & n_{N}=\left.\left(\begin{array}{l}
-y_{\xi} \\
x_{\xi}
\end{array}\right) \frac{1}{\sqrt{x_{\xi}^{2}+y_{\xi}^{2}}}\right|_{\partial \Omega_{N}} .
\end{array}
$$

We introduce the unit normal on the $k$ th cell face $\bar{n}_{k}$ in the physical space, and $\bar{s}_{k}$, in absolute value equal to the length of $\partial \Omega_{k}$. The $\bar{n}_{k}$ and $\bar{s}_{k}$ are defined by

$$
\bar{n}_{k}=\left\{\begin{array}{ll}
n_{k}, & k=E, N, \\
-n_{k}, & k=W, S .
\end{array} \quad \bar{s}_{k}= \begin{cases}s_{k}, & k=E, N, \\
-s_{k}, & k=W, S .\end{cases}\right.
$$


Note that this gives $\bar{n}_{k} \bar{s}_{k}=n_{k} s_{k}, \forall k \in D$. We also introduce the vector $w \in X_{a}^{2} \times E$, of length $2 d+2$. given by

$$
w\left(q, q^{\prime} \cdot n\right)=\left(q, q^{\prime}, \bar{n}\right),
$$

where $q . q^{\prime} \in \mathcal{X}_{12}$ and $\pi \in E$. For the $k$ th cell face we define

$$
\begin{aligned}
u_{k} & =\left(q_{k}, q_{k}, \bar{n}_{k}\right), \\
u_{k}^{L R} & =\left(q_{k}^{L} \cdot q_{k}^{R}, \bar{n}_{k}\right) .
\end{aligned}
$$

Assume that the following, usual conditions hold

$$
\begin{aligned}
F\left(q . q^{\prime} \cdot-n\right) & =-F\left(q, q^{\prime}, n\right) . & & \forall q, q^{\prime} \in X_{a}, \quad n \in E, \\
F(q . q . n) & =f^{*}\left(q^{*}\right) . & & \forall q \in X_{a}, \quad n \in E .
\end{aligned}
$$

The local discretisation error due to the reconstruction can now be written as

$$
\tau_{r}=\frac{1}{A} \sum_{k \in D}\left(F\left(q_{k}^{L}, q_{k}^{R} \cdot n_{k}\right)-f^{*}\left(q_{k}^{*}\right)\right) s_{k} .
$$

With (4.19) and (4.21) we now have

$$
F\left(w_{k}\right)= \begin{cases}f^{*}\left(q_{k}^{*}\right), & k=E . N . \\ -f^{*}\left(q_{k}^{*}\right), & k=W, S .\end{cases}
$$

and with (4.18), (4.22) can be written as

$$
\tau_{r}=\frac{1}{A} \sum_{k \in D}\left(F\left(w_{k}^{L R}\right) \bar{s}_{k}-f^{*}\left(q_{k}^{*}\right) s_{k}\right) .
$$

We denote the reconstruction error $r_{k}$, for the $k$ th cell face by

$$
r_{k}=w_{k}^{L R}-w_{k} \text {. }
$$

We denote the difference between $\left(w_{0}\right)_{k}$ and the mean $w_{k}$ on $\partial \Omega_{k}$ by

$$
\Delta_{k}=w_{k}-\left(w_{0}\right)_{k},
$$

where $\left(w_{0}\right)_{k}$ is defined by

$$
\left(w_{0}\right)_{k}=\left(q_{0}, q_{0},\left(\bar{n}_{0}\right)_{k}\right)
$$

and $\left(\bar{n}_{0}\right)_{k}$ defined by

$$
\left(\bar{n}_{0}\right)_{k}= \begin{cases}\left(\begin{array}{l}
y_{2} \\
-x_{2}
\end{array}\right) \frac{1}{\sqrt{x_{2}^{2}+y_{2}^{2}}}, & k=E, W, \\
\left(\begin{array}{l}
-y_{1} \\
x_{1}
\end{array}\right) \frac{1}{\sqrt{x_{1}^{2}+y_{1}^{2}}}, & k=N, S .\end{cases}
$$

Now, first making a Taylor series expansion of $F(w)$ around the mean $w_{k}$ along cell face $\partial \Omega_{k}$ and substitution of it in (4.23) and next, substitution of an expansion of $F(w)$ around $\left(w_{0}\right)_{k}$, gives

$$
\begin{aligned}
\tau_{r}= & \frac{1}{A} \sum_{k \in D}\left\{\left.\frac{\partial F}{\partial w^{\alpha}}\right|_{\left(w_{0}\right)_{k}} r_{k}^{\alpha}+\left.\frac{\partial^{2} F}{\partial w^{\alpha} \partial w^{\beta}}\right|_{\left(w_{0}\right)_{k}} r_{k}^{\alpha}\left(\Delta_{k}^{\beta}+\frac{1}{2} r_{k}^{\beta}\right)\right. \\
& \left.+\left.\frac{1}{2} \frac{\partial^{3} F}{\partial w^{\alpha} \partial w^{\beta} \partial w^{\gamma}}\right|_{\left(w_{0}\right)_{k}} r_{k}^{\alpha}\left(\Delta_{k}^{\beta} \Delta_{k}^{\gamma}+r_{k}^{3} \Delta_{k}^{\gamma}+\frac{1}{3} r_{k}^{\beta} r_{k}^{\gamma}+\ldots\right)+\ldots\right\} \bar{s}_{k} .
\end{aligned}
$$

Here, again the summation convention is used for $\alpha, \beta$ and $\gamma$. We use (4.25) to study the requirements for consistency. 


\subsection{Consistency requirements}

Now we can formulate the requirements to be satisfied in the reconstruction phase in order to obtain a $p$ th-order consistent or weakly consistent discretisation.

\section{Consistency}

We consider the contributions due to the cell faces $\partial \Omega_{k}, k=E, W$. Assume that the following asymptotic relations hold for $p, q, s=1,2$, and for $r_{k}$ and $\delta_{k}$ as defined by (4.24):

$$
\begin{aligned}
r_{E} & =\mathcal{O}\left(h^{q}\right), \\
r_{W} & =r_{E}+\mathcal{O}\left(h^{r}\right), \quad r \geq q, \\
\Delta_{E} & =\mathcal{O}\left(h^{s}\right), \\
\Delta_{W} & =\Delta_{E}+\mathcal{O}\left(h^{t}\right), \quad t \geq s .
\end{aligned}
$$

For the mapping $M$ we also have

$$
\begin{aligned}
s_{E} & =\mathcal{O}(h), \\
s_{W} & =s_{E}+\mathcal{O}\left(h^{2}\right) .
\end{aligned}
$$

With (4.26) and (4.27), and by changing the order of summation, we find for the first term of $(4.25)$,

$$
\begin{aligned}
r_{E} \bar{s}_{E}+r_{W} \bar{s}_{W} & =r_{E} s_{E}+\left(r_{E}+\mathcal{O}\left(h^{r}\right)\right)\left(-\bar{s}_{E}+\mathcal{O}\left(h^{2}\right)\right) \\
& =\mathcal{O}\left(h^{q+2}\right)+\mathcal{O}\left(h^{r+1}\right) .
\end{aligned}
$$

Hence, a $p$ th-order consistent discretisation requires

$$
\begin{aligned}
q & \geq p \\
r-1 & \geq p
\end{aligned}
$$

It can easily be shown that all other terms in (4.25) give an $\mathcal{O}\left(h^{p}\right)$ or smaller contributions to $\tau_{r}$, provided $p, q, s \geq 1$ and (4.28) are satisfied.

\section{Weak consistency}

For weak consistency and its requirements with respect to reconstruction, we consider a cell $\Omega_{i, j}^{l}$ and all its descendants when the composite grid is $n$ times refined. The definitions of the previous subsections hold, but a superscript specifying the level and a subscript for the cell number are added. The superscript $n$ denotes level $l+n$, subscript $r$ denotes cell index $2^{n} i+r$ and subscript $s$ denotes index $2^{n} j+s$. The collective local discretisation error is now given by

$$
\left\{T_{n}^{l}(q)\right\}_{i, j}^{l}=\frac{1}{A_{i, j}^{l}} \sum_{r, s=0}^{2^{n}-1} A_{r, s}^{n} \tau_{r, s}^{n}(q) .
$$

With $\tau^{l}(q)=\tau_{q}^{l}(q)+\tau_{r}^{l}(q)$, we have

$$
A_{r, s}^{n} \tau_{r, s}^{n}(q)=\sum_{k \in D}\left(F\left(\left(w^{L R}\right)_{r, s, k}^{n}\right) \bar{s}_{r, s, k}^{n}-f_{r, s, k}^{* n}(q) s_{r, s, k}^{n}\right)
$$


Assume the numerical flux $F\left(\left(w^{L R}\right)_{r, s, k}^{n}\right)$ across cell face $\partial \Omega_{r, s, k}^{n}$ to be an $\mathcal{O}\left(h_{l+n}^{q}\right)$ accurate approximation of the mean flux $f_{r . s . k}^{* n}(q)$. We have

$$
F\left(\left(w^{L R}\right)_{r, s, k}^{n}\right) \bar{s}_{r, s, k}^{n}=f_{r, s, k}^{* n}(q) s_{r, s, k}^{n}+\mathcal{O}\left(h_{l+n}^{q+1}\right) .
$$

Substitution of (4.31) and (4.30) into (4.29) yields a summation over all cell faces on the 'outer' boundary $\partial \Omega_{r, s . k}^{n} \subset \partial \Omega_{i, j, k}^{l}, \forall k \in D$. We consider $k=W$ and $n \rightarrow \infty$. For the contribution of the approximations on these cell faces to $\left\{T_{n}^{l}\right\}_{i, j}^{l}$ we find with (4.31)

$$
\begin{aligned}
& \frac{1}{A_{i, j}^{l}} \sum_{s=0}^{2^{n}-1}\left(F\left(\left(w^{L R}\right)_{0, s, W}^{n}\right) \bar{s}_{0, s, W}^{n}-f_{0, s, W}^{* n}(q) s_{0 . s . W}^{n}\right) \\
& \quad=\frac{1}{A_{i, j}^{l}} \sum_{s=0}^{2^{n}-1}\left(C_{1} h_{l+n}^{q+1}+\mathcal{O}\left(h_{l+n}^{q+2}\right)\right) \\
& \quad=C_{2} h_{l+n}^{q}+\mathcal{O}\left(h_{l+n}^{q+1}\right),
\end{aligned}
$$

where $C_{1}$ and $C_{2}$ are constants independent of the level $n$. Similar results hold for $k=N, E, S$. Hence, if we have an $\mathcal{O}\left(h_{l+n}^{q}\right)$ accurate approximation of the mean value along a cell face of the flux across the cell face, then the collective local discretisation error is $\mathcal{O}\left(h_{l+n}^{q}\right)$ and hence. the approximation is $q$ th-order weakly consistent. So, a weakly consistent approximation of order $p=1,2$, is obtained by $p$ th-order accurate approximation of the mean flux. Let the reconstruction error be defined by (4.24). By the fact that the quadrature rule yields a second-order contribution, and by expansion of $F(w)$ around the mean $w_{r, s, k}^{l+n}$, it follows that a reconstruction error of order $p=1,2$, yields a $p$ th-order weakly consistent discretisation.

Note that consistency of order $p=1,2$, implies weak consistency, since a necessary but not sufficient condition for consistency is a reconstruction error of order $p$, as given by $(4.28 \mathrm{a})$.

\section{$5 \quad$ Interpolations for virtual states}

In this section we study a procedure for the computation of the virtual states. We consider a grid in the physical domain obtained by an affine mapping $M^{l}=M$. An analysis for more general mappings would probably require an invariant description in general coordinates, similar as e.g. in [11]. We derive expressions for the error of the discretisation due to the computation of the virtual states. For an affine mapping $M$ and a function $q$ we have

$$
q_{i, j}^{l}=\left\{\bar{R}_{q}^{l} q\right\}_{i, j}^{l}=\frac{1}{h_{l}^{2}} \int_{\tilde{\Omega}_{i, j}^{l}} q d \tilde{\Omega},
$$

This simplifies the analysis, since we can express all in computational $(\xi, \eta)$ coordinates. We first establish the accuracy of the virtual states for first and second-order accurate discretisations.

We assume a sufficiently smooth function $q$, and a Taylor series expansion, given by

$$
\begin{aligned}
q= & q_{0}+q_{1} \xi+q_{2} \eta+q_{3} \xi^{2}+q_{4} \xi \eta+q_{5} \eta^{2} \\
& +q_{6} \xi^{3}+q_{7} \xi^{2} \eta+q_{8} \xi \eta^{2}+q_{9} \eta^{3}+\ldots
\end{aligned}
$$

around the centre of the cell $\Omega_{i, j}^{l}$. We consider the equations for a cell $\Omega_{2 i+1,2 j+1}^{l+1}$, where $\partial \Omega_{2 i+1,2 j+1, E}^{l+1}$ is part of the green boundary on level $l+1$. The virtual state $v_{2 i+2,2 j+1}^{l+1}$ 
required for these equations, is an approximation of the mean of $q$ on $\omega_{2 i+2,2 j+1}^{l+1}$. This mean can be expressed as

$$
\begin{aligned}
& \frac{1}{h_{l+1}^{2}} \int_{\bar{\omega}_{2+1}^{l+2 j+1}} q d \tilde{\Omega}=q_{0}+\frac{3}{2} h_{l+1} q_{1}+\frac{1}{2} h_{l+1} q_{2} \\
& \quad+\frac{7}{3} h_{l+1}^{2} q_{3}+\frac{3}{4} h_{l+1}^{2} q_{4}+\frac{1}{3} h_{l+1}^{2} q_{5}+\frac{15}{4} h_{l+1}^{3} q_{6} \\
& \quad+\frac{3}{6} h_{l+1}^{3} q_{7}+\frac{1}{2} h_{l+1}^{3} q_{8}+\frac{1}{4} h_{l+1}^{3} q_{9}+\mathcal{O}\left(h_{l+1}^{4}\right)
\end{aligned}
$$

This equals $q_{2 i+2,2 j+1}^{l+1}$ in the situation $(2 i+2.2 j+1) \in I^{l}$, since $\omega_{2 i+2,2 j+1}^{l+1}$ is the part of $\widehat{\Omega}$ that would be $\Omega_{2 i+2,2 j+1}^{i+1}$ if the grid would be sufficiently refined (cf. (3.13)). In [17] it is shown that $q_{2 i+2,2 j+1}^{l+1}$ (and hence (5.1)) satisfies the requirements for consistency (4.28), for the reconstructions discussed in Section 3.4. For any virtual state $v_{2 i+2,2 j+1}^{l+1}$ that differs $\mathcal{O}\left(h_{l+1}^{p}\right), p=1,2$ from (5.1), the reconstructions discussed in Section 3.4 do not satisfy the consistency requirements and introduce an $\mathcal{O}\left(h_{l+1}^{p-1}\right)$ error in the equations for $\Omega_{2 i+1,2 j+1}^{l+1}$. However, the numerical flux will be a $p$ th-order accurate approximation of the mean value of the exact flux, and hence pth-order consistency in the weak sense is obtained (cf. 4.5).

\subsection{Virtual states for weak consistency}

For $p$ th-order weak consistency we only require a $p$ th-order accurate approximation of the mean flux across a cell face. We consider virtual states for both first and second-order weak consistency.

\section{First-order}

The formula for a first-order accurate virtual state $v_{2 i+2,2 j+1}^{l+1}$ is

$$
v_{2 i+2,2 j+1}^{l+1}=q_{i+1, j}^{l}
$$

For this virtual state we find with the Taylor series expansion

$$
v_{2 i+2,2 j+1}^{l+1}=q_{0}+2 h_{l+1} q_{1}+\mathcal{O}\left(h_{l+1}^{2}\right) \text {. }
$$

This differs $\mathcal{O}\left(h_{l+1}\right)$ from (5.1). Hence, it yields a zeroth-order error for the equations. However, since the virtual state is $\mathcal{O}\left(h_{l}\right)$ accurate, the reconstruction gives a first-order accurate virtual state, which yields a flux computation which is first-order accurate. This implies first-order weak consistency.

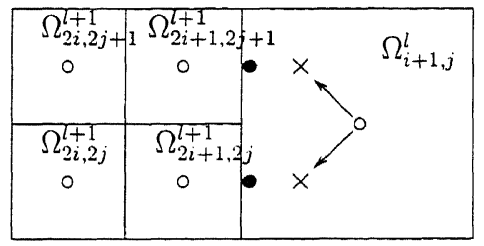

Figure 5.1: Virtual state for first-order weak consistency on a locally refined grid; 0 : available state; $\bullet$ : left or right state; $\times$ : virtual state. 


\section{Second-order}

A similar situation for second-order weak consistency is found, if the virtual states are computed with the second-order accurate formulas

$$
\begin{aligned}
& v_{2 i+2,2 j+1}^{l+1}=\frac{3}{4} q_{i+1, j}^{l+1}+\frac{1}{4} q_{i, j+1}^{l+1}, \\
& v_{2 i+3,2 j+1}^{l+1}=\frac{3}{4} q_{i+1, j}^{l+1}+\frac{1}{4} q_{i+2, j+1}^{l+1} .
\end{aligned}
$$

Again, the virtual state $v_{2 i+2,2 j+1}^{l+1}$ is an approximation of the mean of $q$ on $\omega_{2 i+2,2 j+1}^{l+1}$, given in (5.1). For the virtual state computed in this way, we have

$$
\begin{aligned}
v_{2 i+2,2 j+1}^{l+1}= & q_{0}+\frac{3}{2} h_{l+1} q_{1}+\frac{1}{2} h_{l+1} q_{2}+\frac{10}{3} h_{l+1}^{2} q_{3} \\
& +\frac{4}{3} h_{l+1}^{2} q_{5}+\mathcal{O}\left(h_{l+1}^{3}\right) .
\end{aligned}
$$

A similar result is obtained for $v_{2 i+3,2 j+1}^{l+1}$. Apparently, this is a second-order accurate approximation of (5.1). Similar to the first-order weakly consistent situation, the consistency requirements (4.28) are not satisfied for $p=2$. However, the flux is second-order accurate, hence (5.4) yield second-order weak consistency. These formulas are two of a number of possible choices for second-order accurate virtual states. We have chosen these for their relative compactness and their symmetry. They are symmetric with respect to the diagonal through the centres of $\Omega_{i, j+1}^{l}$ and $\Omega_{i+1, j}^{l}$. A virtual state required for e.g. $\Omega_{2 i+2,2 j+2}^{l+1}$ with $\partial \Omega_{2 i+2,2 j+2, S}$, which is a part of the green boundary on level $l+1$, exactly results in (5.4a).

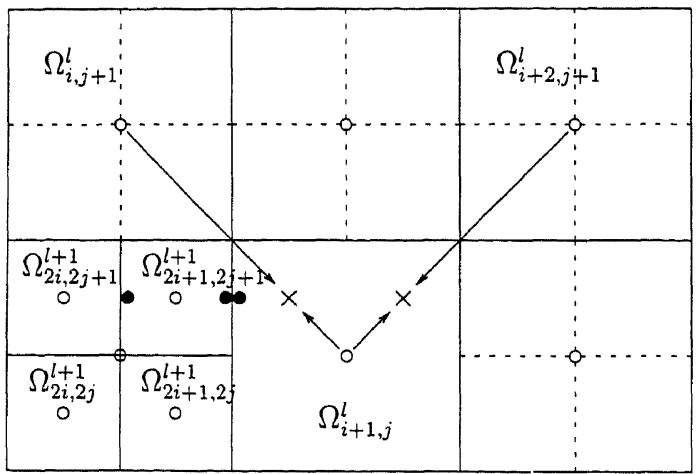

Figure 5.2: Virtual states for second-order weak consistency and first-order consistency on a locally refined grid; 0 : available state; $\bullet$ : left or right state; $\times$ : virtual state.

\subsection{Virtual states for consistency}

The requirements to be satisfied for a consistent discretisation, are given by (4.28). We consider both first and second-order consistency. A $p$ th-order consistent discretisation for equations for cells near green boundaries requires a $(p+1)$ st accurate computation of virtual states. 


\section{First-order}

First-order consistency can be obtained by second-order accurate computation of virtual states. For this the formulas (5.4) are applied. From (5.1) and (5.5) it is clear that the requirements (4.28) are satisfied for $p=1$, if they are satisfied by $q_{2 i+2,2 j+1}^{l+1}$ when $\Omega_{2 i+2,2 j+1}^{l+1}$ would exist. This is shown to be the case in [17].

\section{Second-order}

Similarly a third-order accurate computation of the virtual state is required for secondorder consistency. This causes no extra second-order error with respect to the situation where $\Omega_{2 i+2.2 j+1}^{l+1}$ would exist. As shown in [17], in that situation (i.e. $\Omega_{2 i+2,2 j+1}^{l+1}$ exists) a second-order accurate discretisation is obtained.

A third-order accurate computation of virtual states is given by

$$
\begin{aligned}
v_{2 i+2,2 j+1}^{l+1}= & \frac{17}{16} q_{i+1, j}^{l}+\frac{1}{16}\left(q_{i, j}^{l}+q_{i, j+1}^{l}+q_{i+1, j+1}^{l}\right) \\
& -\frac{2}{16}\left(q_{i+2, j}^{l}+q_{i+1, j-1}^{l}\right), \\
v_{2 i+3,2 j+1}^{l+1}= & \frac{17}{16} q_{i+1 . j}^{l}+\frac{1}{16}\left(q_{i+2, j}^{l}+q_{i+2, j+1}^{l}+q_{i+1, j+1}^{l}\right) \\
& -\frac{2}{16}\left(q_{i, j}^{l}+q_{i+1, j-1}^{l}\right) .
\end{aligned}
$$

This is schematically represented in Fig. 5.3.

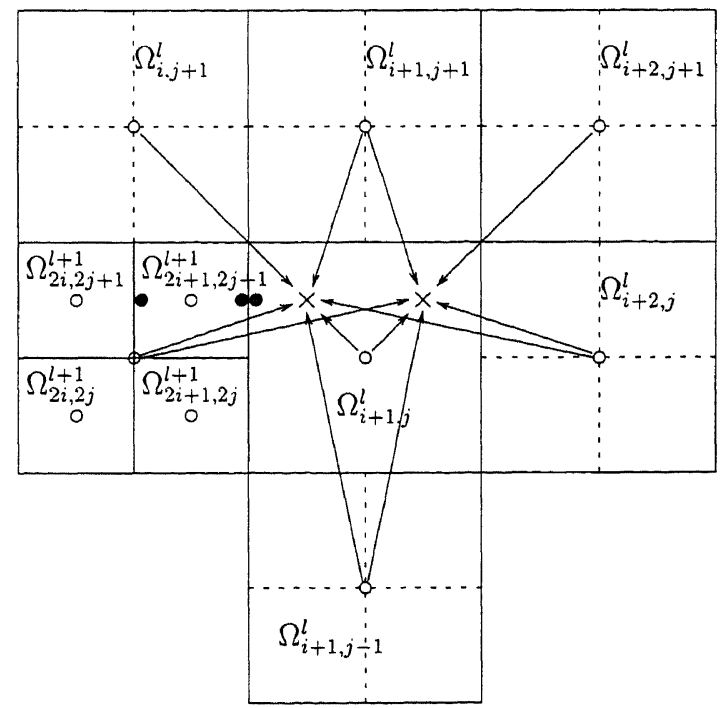

Figure 5.3: Virtual states for second-order consistency on a locally refined grid; 0 : available state; $\bullet$ : left or right state; $\times$ : virtual state.

These are also chosen from a number of possible alternatives. Apart from compactness and symmetry, this choice is based on the size of the in absolute value largest negative coefficients. For the present choice, the negative coefficients are smaller in absolute value than for possible alternatives with similar compactness and symmetry. From the Taylor 
series expansion of $q$ it can be shown that the virtual state $v_{2 i+2,2 j+1}^{l+1}$, obtained by (5.6a) can be expressed as

$$
\begin{aligned}
v_{2 i+2.2 j+1}^{l+1}= & q_{0}+\frac{3}{2} h_{l+1} q_{1}+\frac{1}{2} h_{l+1} q_{2}+\frac{7}{3} h_{l+1}^{2} q_{3}+\frac{3}{4} h_{l+1}^{2} q_{4} \\
& +\frac{1}{3} h_{l+1}^{2} q_{5}+\frac{3}{2} h_{l+1}^{3} q_{6}+\frac{5}{3} h_{l+1}^{3} q_{7}+3 h_{l+1}^{3} q_{9}+\mathcal{O}\left(h_{l+1}^{4}\right) .
\end{aligned}
$$

Clearly this is a third-order accurate approximation of the mean value of $q$ on $\omega_{2 i+2,2 j+1}^{l+1}$. Hence. since this virtual state does not introduce additional second-order errors in the reconstruction. the requirements for consistency (4.28) are satisfied for $p=2$ and the discretisation is second-order consistent.

\section{References}

[1] T.J. Barth and P.O. Frederickson. Higher order solution of the Euler equations on unstructered grids, using quadratic reconstruction. In AIAA-90-0013, 1990.

[2] T.J. Barth and D.C. Jespersen. The design and application of upwind schemes on unstructured meshes. In AIAA-89-0366. 1989.

[3] A. Brandt. Multi-level adaptive techniques (MLAT) for singular perturbationproblems. In P.W. Hemker and J.J.H. Miller, editors, Numerical Analysis of Singular Perturbation Problems, pages 53-142. Academic Press, 1979.

[4] S.K. Godunov. Finite difference method for numerical computation of discontinuous solutions of the equations of fluid dynamics. Math. Sbornik, 47:271-306, 1959. Translated from Russian: Cornell Aeronautical Laboratory.

[5] W. Hackbusch. Multi-Grid Methods and Applications, volume 4 of Springer Series in Computational Mathematics. Springer-Verlag, Berlin, 1985.

[6] W. Hackbusch and U. Trottenberg, editors. Multigrid Methods II, Proc. of the 2nd European Conference on Multigrid Methods, held in Cologne, 1985, Lecture Notes in Mathematics, vol. 1228. Springer-Verlag, 1986.

[7] P.W. Hemker. Defect correction and higher order schemes for the multi grid solution of the steady Euler equations. In Hackbusch and Trottenberg [6], pages 149-165.

[8] P.W. Hemker and S.P. Spekreijse. Multiple grid and Osher's scheme for the efficient solution of the steady Euler equations. Appl. Num. Math., 2:475-493, 1986.

[9] B. Koren. Defect correction and multigrid for the efficient and accurate computation of airfoil flows. J. Comput. Phys., 77:183-206, 1988.

[10] P.D. Lax. Hyperbolic Systems of Conservation Laws and the Mathematical Theory of Shock Waves, volume 11 of Regional Conference Series in Applied Mathematics. Society for Industrial and Applied Mathematics, Philadelphia, 1973.

[11] C.W. Oosterlee and P. Wesseling. A multigrid method for an invariant formulation of the incompressible Navier-Stokes equations in general coordinates. Comm. Appl. Numer. Methods, 8:721-734, 1992. 
[12] S. Osher and F. Solomon. Upwind difference schemes for hyperbolic systems of conservation laws. Math. Comp., 38(158):339-374, 1982.

[13] P.L. Roe. Approximate Riemann solvers, parameter vectors and difference schemes. J. Comput. Phys., 43:357-372, 1981.

[14] G.H. Schmidt and F.J. Jacobs. Adaptive local grid refinement and multi-grid in numerical reservoir simulation. J. Comput. Phys., 77:140-165. 1988.

[15] S.P. Spekreijse. Multigrid solution of monotone second-order discretizations of hyperbolic conservation laws. Math. Comp., 49(179):135-155, 1986.

[16] S.P. Spekreijse. Second order accurate upwind solutions of the 2D steady Euler equations by the use of a defect correction method. In Hackbusch and Trottenberg [6], pages 285-300.

[17] S.P. Spekreijse. Multigrid Solution of the Steady Euler Equations. CWI. Amsterdam. 1988. CWI-tract 46.

[18] J.L. Steger and R.F. Warming. Flux vector splitting in the inviscid gasdynamic equations with application to finite-difference methods. J. Comput. Phys., 10:263$293,1981$.

[19] P.K. Sweby. High resolution schemes using flux limiters for hyperbolic conservation laws. SIAM J. Numer. Anal., 21:995-1011, 1984.

[20] H.T.M. van der Maarel. A Local Grid Refinement Method for the Euler Equations. $\mathrm{PhD}$ thesis, University of Amsterdam, 1993. To appear as CWI Tract, CWI, Amsterdam, 1994.

[21] B. van Leer. Towards the ultimate conservative difference scheme. IV. A new approach to numerical convection. J. Comput. Phys., 23:276-299, 1977.

[22] B. van Leer. Flux-vector splitting for the Euler equations. In E. Krause, editor, Proceedings of the Eight International Conference on Numerical Methods in Fluid Dynamics, Aachen, 1982, Lecture Notes in Physics, vol. 170, pages 507-512. SpringerVerlag, 1982.

[23] B. van Leer. Upwind difference methods for aerodynamic problems governed by the Euler equations. In B.E. Engquist, S.J. Osher, and R.C.J. Sommerville, editors, Large Scale Computations, Lectures in Applied Mathematics part 2, pages 327-336. AMS, 1985.

[24] B. van Leer. Towards the ultimate conservative difference scheme. V. A second order sequel to Godunov's method. J. Comput. Phys., 32:101-136, 79. 